\title{
BEACH AND INDOOR VOLLEYBALL ATHLETES PRESENT SIMILAR LOWER LIMB MUSCLE ACTIVATION DURING A COUNTERMOVEMENT JUMP
}

original paper

(1) University School of Physical Education in Wroclaw

DOI: https://doi.org/10.5114/hm.2020.89913

\author{
FABIO DAL BELLO ${ }^{1,2}$, ESTEBAN AEDO-MUÑOZ ${ }^{1,3}$, DANILO GOMES MOREIRA ${ }^{4}$, \\ CIRO JOSÉ BRITO ${ }^{5}$, BIANCA MIARKA ${ }^{6}$, ENRIQUE NAVARRO CABELLO ${ }^{7}$ \\ ${ }^{1}$ Magíster en Ciencias de la Actividad Física y Deporte, Facultad de Salud, Universidad Santo Tomás, Santiago, Chile \\ ${ }^{2}$ Polytechnic University of Madrid, Madrid, Spain \\ ${ }^{3}$ Physical Activity, Sport and Health Sciences Laboratory, University of Santiago, Santiago, Chile \\ ${ }^{4}$ Federal Institute of Minas Gerais, Brazil \\ ${ }^{5}$ Physical Education Department, Federal University of Juiz de Fora, Juiz de Fora, Brazil \\ ${ }^{6}$ School of Physical Education and Sports, Federal University of Rio de Janeiro \\ ${ }^{7}$ Sports Biomechanics Laboratory, Polytechnic University of Madrid, Madrid, Spain
}

\begin{abstract}
Purpose. The study aimed to compare and correlate the power, height, eccentric and concentric force development rate of 3 sequential attempts of countermovement jump (CMJ) and the respective muscle response in beach and indoor volleyball athletes.

Methods. The sample involved high-level hard court indoor volleyball (HCIV, $n=7$ ) and high-level sand court beach volleyball (SCBV, $n=6)$ athletes. They performed $3 \mathrm{CMJs}\left(1^{\text {st }} \mathrm{CMJ}\right.$ vs. $2^{\text {nd }} \mathrm{CMJ}$ vs. $\left.3^{\text {rd }} \mathrm{CMJ}\right)$ to determine the height, eccentric force development rate (EFDR), concentric force development rate (CFDR), power, and lower limbs muscle electrical activity.

Results. Difference was demonstrated between HCIV vs. SCBV players in $3^{\text {rd }}$ CMJ EFDR $(-270.2 \pm 31.6 \mathrm{~N} / \mathrm{s}$ vs. $-214.3 \pm$ $38.7 \mathrm{~N} / \mathrm{s})$. In HCIV, $1^{\text {st }} \mathrm{CMJ}$ was different from $2^{\text {nd }} \mathrm{CMJ}$ and $3^{\text {rd }} \mathrm{CMJ}$ in EFDR $(-239.0 \pm 27.3 \mathrm{~N} / \mathrm{s}$ vs. $-285.6 \pm 40.2 \mathrm{~N} / \mathrm{s}$ and $-270.2 \pm 31.6 \mathrm{~N} / \mathrm{s})$, CFDR $(87.8 \pm 24.4 \mathrm{~N} / \mathrm{s}$ vs. $89.6 \pm 25.7 \mathrm{~N} / \mathrm{s}$ and $75.0 \pm 23.6 \mathrm{~N} / \mathrm{s})$, and power $(2341.8 \pm 342.3 \mathrm{~W}$ vs. $2433.9 \pm 327.2 \mathrm{~W}$ and $2411.0 \pm 358.5 \mathrm{~W})$. In SCBV, $2^{\text {nd }} \mathrm{CMJ}$ was different from $3^{\text {rd }} \mathrm{CMJ}$ in EFDR $(-267.1 \pm 45.2 \mathrm{~N} / \mathrm{s}$ vs. $-214.3 \pm 38.7 \mathrm{~N} / \mathrm{s}$ ). In $2^{\text {nd }} \mathrm{CMJ}$, the left gluteus presented lower electrical activity than the left medial gastrocnemius, and HCIV revealed a difference between the response of the right and left gluteus in $3^{\text {rd }} \mathrm{CMJ}$.

Conclusions. Our analysis demonstrated ca. $70 \%$ of good or excellent intra-class correlation between the beach and indoor groups.
\end{abstract}

Key words: countermovement jump, volleyball biomechanics, EMG, muscle activity

\section{Introduction}

Indoor and beach volleyball have been part of the official program of the Summer Olympic Games, despite their similarities. Apart from the obvious difference between hot sand and hard court, there are factors to consider regarding the transitions from beach to indoor volleyball, especially the jump power [1]. The capacity of the neuromuscular system to produce power using the lower limbs is critical for performance in beach and indoor volleyball, since in both there are two teams separated by a net trying to score points by grounding a ball on the other team's court with actions that involve jumping activities such as serving, attacking, blocking, and setting [1-3]. The average of beach and indoor rallies per set is ca. $30 \mathrm{~s}$ and ca. $45 \mathrm{~s}$, with a work-to-rest ratio of 1:4 and 1:5, respectively $[4,5]$, considering that the player has up to $8 \mathrm{~s}$ to draw the ball and that each player jumps and touches the ball at least once per set and minute $[4,5]$. Thus, it would be interesting to check the difference between maximal vertical jumping performed by the use of a sequential dynamic strategy, with a gap of 1-min rest, similar to what occurs during the game $[4,5]$. To do so,

Correspondence address: Fabio Dal Bello, Magíster en Ciencias de la Actividad Física y Deporte,

Universidad Santo Tomás, Av. Ejercito, 146, Santiago, Chile, e-mail: fabaoquiro@hotmail.com

Received: April 10, 2019

Accepted for publication: August 21, 2019

Citation: Dal Bello F, Aedo-Muñoz E, Moreira DG, Brito CJ, Miarka B, Cabello EN. Beach and indoor volleyball athletes present similar lower limb muscle activation during a countermovement jump. Hum Mov. 2020;21(2):42-50; doi: https:// doi.org/10.5114/hm.2020.89913. 
the countermovement jump (CMJ) is a useful physical performance test to evaluate the athletic potential of volleyball players owing to its good or excellent positive correlation with speed, power, agility, and strength performance [6].

$\mathrm{CMJ}$ allows potential energy to be converted to downward kinetic energy and then elastic strain energy [6]. During a maximal vertical jump starting from a static preparatory position, $\mathrm{CMJ}$ is performed with no other constraints than the anatomy, and it seems likely that the use of different strategies is solely dependent on the subject's particularities [6]. Therefore, it is possible to verify the differences between beach and indoor players, which make it possible to understand the effects of training on the sand surface and on the hard court.

In fact, the main differences between indoor and beach volley are in the court conditions (hard vs. sand), as well as in the rules; in a match, beach volleyball consists of a series of best-of-three games, with each game played to 21 points [5]. Two sets win the match, and the third tiebreaker set, if necessary, is also played to 15 points [5]. There is a difficulty of jumping in the sand and performing the necessary actions in the game, while indoor volleyball seems to be longer, as a match consists of 5 sets, or games [4]. The first team to reach 25 points wins the set [4]. Three sets win the match, and the fifth tiebreaker set, if necessary, is only played to 15 points [4]. Therefore, indoor games are longer and less intense, which may interfere with the variables associated with jumping. In contrast, since physical and technical demands tend to increase during the long-term development of the athlete (related to athletes' specialization) [3], it is also expected that the power-related capacities (i.e. vertical jump ability) will simultaneously increase throughout the high-level athletes' preparation processes $[5,6]$ and present differences between the two volleyball modalities. This means that both show direct dependence on neural activation of the lower limbs for jumps and rapid movements during the game, while beach volleyball seems to present more intensity, volume, and apparent muscle activity changes caused by longer rallies, reduced speed and mobility on the beach [3, 4].

Previous studies have compared submaximal and maximal vertical CMJs, in which the effects demonstrate different motor strategies being employed for the 2 situations, with muscle power in such submaximal jumps being mainly generated from the calf muscles, while the mechanical energy for maximal jumps mostly comes from the thigh and hip musculature [6]. Aside from muscle strength and ground reaction force, electromyographic behaviour (i.e. assessment of neuromuscular control) also plays a key role in vertical jump performance [7, 8]. Consequently, it is not known whether electromyography (EMG) parameters describe muscle activity differences between beach and indoor volleyball training, as well as whether the specific training of beach volleyball causes more positive effects on the improvement of motor unit recruitments than indoor training [6-8]. This factor is particularly important because similar magnitude of force and the way it is exerted (i.e. step or ramp contractions) can show that beach or indoor volleyball players could compete in one or another mode with easy adaptation [9-12].

Based on the assumption that jumping is one of the most important actions in the development of a volleyball game (beach or indoor) [3, 4], the knowledge of parameters related to jumping as the variables of time, force, the correlation between both (ratio of development of force, impulse, and power), and the EMG variables may be useful for training planning [13-16]. This information can be obtained by performing and evaluating CMJs and allows coaches and researchers to understand a subject's jumping characteristics, and more specifically the different phases of the movement (eccentric and concentric) [17]. In the context of the technical and tactical differences or similarities in playing beach and indoor volleyball modalities, the aim of this study was to compare and verify correlations between the power, height, eccentric and concentric force development rate of 3 sequential attempts of jumps and the respective muscle electric activity of the gluteus maximus, rectus femoris, vastus medialis, and medial gastrocnemius of left and right lower limbs based on EMG signal during $\mathrm{CMJ}$ in beach and indoor volleyball athletes. We hypothesized that the EMG signal of the analysed muscles would be different for the attempts, specifically indoor volleyball athletes would present higher EMG during the eccentric sub-phase and respective force development rate than the beach volleyball group.

\section{Material and methods}

\section{Experimental approach}

This was a comparative and descriptive applied study using EMG analysis. We aimed to determine specific EMG characteristics of extended muscles of the lower limbs analysed during the $\mathrm{CMJ}$ in beach and indoor volleyball athletes. A validated CMJ protocol was identified in previous studies addressing volley- 
ball jump performance analysis [14, 16]. After this, the data collection from the Chilean High Performance Center was analysed. All CMJ tests were observed in a controlled biomechanics laboratory environment between 10:00 and 12:00 a.m. at the temperature of $24.5-27.0^{\circ} \mathrm{C}$. We subsequently compared the EMG data from CMJ tests of high-level beach and indoor volleyball players. CMJ height $(\mathrm{cm})$, power $(\mathrm{W})$, and ground reaction force $(\mathrm{N})$ were evaluated by using an infrared jump system (Optojump, Microgate ${ }^{\circledR}$, Bolzano, Italy) interfaced with a microcomputer in order to assess the muscle power of the lower limbs $[18,19]$ to obtain ground reaction force data. The gathered information included: jump height $(\mathrm{cm})$, eccentric force development rate (EFDR; N/s), concentric force development rate (CFDR; N/s) (Figure 1), power (W), and muscle activation evaluated by wireless EMG of the gluteus maximus, rectus femoris, vastus medialis, and medial gastrocnemius muscles. The study ensured anonymity and confidentiality by replacing the athletes' personal identification data.

\section{Participants}

The sample was composed of 13 male Chilean international volleyball athletes: high-level hard court indoor volleyball (HCIV) players $(n=7$; age: $25.5 \pm$ 2.8 years; height: $191.1 \pm 1.3 \mathrm{~cm}$; weight: $84.9 \pm 5.1 \mathrm{~kg}$ ) and high-level sand court beach volleyball (SCBV) players ( $n=6$; age: $22.0 \pm 3$ years; height: $188.0 \pm$ $1.4 \mathrm{~cm}$; weight: $83.5 \pm 4.7 \mathrm{~kg}$ ) aged $>18$ years, from the indoor and beach volleyball national teams. All athletes were exclusively beach or indoor players. The sample calculation was performed with a confidence level of $95 \%$ and a margin of error of $15 \%$, with the use of the following equations:

$$
\begin{gathered}
\mathrm{x}=\mathrm{Z}(\mathrm{c} / 100) 2 \mathrm{r}(100-\mathrm{r}) \\
\mathrm{n}=\mathrm{N} \mathrm{x} /[(\mathrm{N}-1) \mathrm{E} 2+\mathrm{x}] \\
\mathrm{E}=\operatorname{Sqrt}[(\mathrm{N}-\mathrm{n}) \mathrm{x} / \mathrm{n}(\mathrm{N}-1)]
\end{gathered}
$$

where $\mathrm{N}$ is the population size, $\mathrm{r}$ is the fraction of responses that one is interested in, and $\mathrm{Z}(\mathrm{c} / 100)$ is the critical value of the confidence level c [20].

The study involved all the Chilean beach volleyball athletes of the national team and $60 \%$ of the indoor volleyball team, and training occurred only on beach and only on hard court indoor, depending on the category. The players participated in international representative competitions 2-4 times per month and were regularly training 5 times per week, 6 hours per day (3 hours of technical-tactical training, 3 hours of physi- cal fitness and specific prophylaxis exercises) for SCBV players, and 3.5 hours per day (3 hours of technicaltactical training, 45 min of physical fitness and specific prophylaxis exercises) for HCIV players. It was recommended that subjects avoided alcohol consumption at least 72 hours prior to the test and during protocol, and maintained normal diets. During the preceding 6 months and at the moment of the evaluation, the participants were free of any injury that would limit their ability to perform CMJ. A description of the protocol and a demonstration of the testing procedures were provided to the participants prior to completing any laboratory activities.

\section{Procedures}

The athletes completed two laboratory sessions separated by 48 hours. The first session aimed to obtain written consent and allow the participants to familiarize themselves with the CMJ performance within the laboratory environment, do the jump protocol, and perform 2 sub-maximum jumps 48 hours before the evaluation [18]. During the first session, all test procedures were explained to the subjects.

We performed screening of health risks and obtained informed consents, prepared forms and recorded basic information such as age, height, body weight, gender, test conditions. We checked and calibrated the timing infrared jump system measurement. All subjects performed an appropriate warm-up, with the procedure similar to that in the second session, used to measure the $3 \mathrm{CMJ}$ attempts. Jump height was calculated by using a timing infrared jump system (Optojump, Microgate ${ }^{\circledR}$, Bolzano, Italy), which measures the time of the feet being off the floor. The athlete stands upright in socks or barefoot, as still as possible on the floor, with weight evenly distributed between both feet. Hands are placed on the hips throughout the test. When all is ready, the athlete squats down until the knees are bent at $90^{\circ}$, then immediately jumps vertically as high as possible, landing back on the floor on both feet at the same time [7, 8]. The present study allowed a minimum of 5 min of passive rest between trials, following preceding suggestions [21]. During CMJ, the take-off must be from both feet, with no initial steps or shuffling. Athletes must also not pause at the base of the CMJ.

In the second session, the subjects were evaluated in a single measurement of $3 \mathrm{CMJ}$ attempts. They initially performed a warm-up that consisted of $10 \mathrm{~min}$ on a static cycloergometer at $50-60 \%$ of heart rate and $10 \mathrm{~min}$ of stretching, as described in previous 
studies [22-25]. All stretches were taken to the "pain threshold' as indicated by the subject so that the muscle-tendon system was stretched to its limit without pain, following previous protocols [26]. The evaluation procedure consisted of each subject performing 3 jumps with 1 min of rest between them; the applied recovery time was similar to that between one jump and another during rallies, i.e. ca. 1 min [3, 4]. CMJ height $(\mathrm{cm})$ and power $(\mathrm{W})$ were evaluated by using an infrared jump system (Optojump, Microgate ${ }^{\circledR}$, Bolzano, Italy) interfaced with a microcomputer in order to assess the muscle power of the lower limbs [19] to obtain ground reaction force $(\mathrm{N})$ data by second $(\mathrm{s})$.

\section{Electromyography data acquisition}

Surface EMG data were obtained synchronously with the reaction force data by using a 16-channel acquisition system (Delsys Trigno Wireless FAQ System, USA). An 8-channel protocol was applied, gluteus maximus, rectus femoris, vastus medialis, and medial gastrocnemius muscles of the left and right lower limbs, with the peak value of EMG for each muscle group. Analog signals were interfaced to a personal computer running the EMGworks software ${ }^{\circledast}$ system (Delsys Trigno Wireless FAQ System, USA). Trigno EMG sensors employed 4 silver bar contacts for detecting the EMG signal at the skin surface. For maximum signal amplitude, the bars were oriented perpendicularly to the muscle fibre direction. The top of each sensor was shaped with an arrow to aid in determining the orientation. The sensor was placed in the centre of the muscle belly, away from tendons and the muscle edge. The sensor was attached to the skin with the Delsys Adhesive Sensor Interface (Delsys Trigno Wireless FAQ System, USA). The Trigno system provided simultaneous analogue signal data reconstruction detected by all active sensors. The signals were made available on the 68-pin connectors located on the base station. EMG signals at these outputs were amplified by a factor of 909 , with full dynamic range of $\pm 5 \mathrm{~V}$.

For the location of the EMG sensors, the Surface ElectroMyoGraphy for the Non-Invasive Assessment of Muscles recommendations [27] were applied; after the sensor was inserted, for each muscle evaluated, the subject was asked to generate the largest contraction force possible for 10 seconds against resistance. Therefore, the maximum voluntary muscle contraction was carried out in each evaluated muscle group to establish the real muscle electrical activity in 100\% of activation in all the subjects, and visual execution

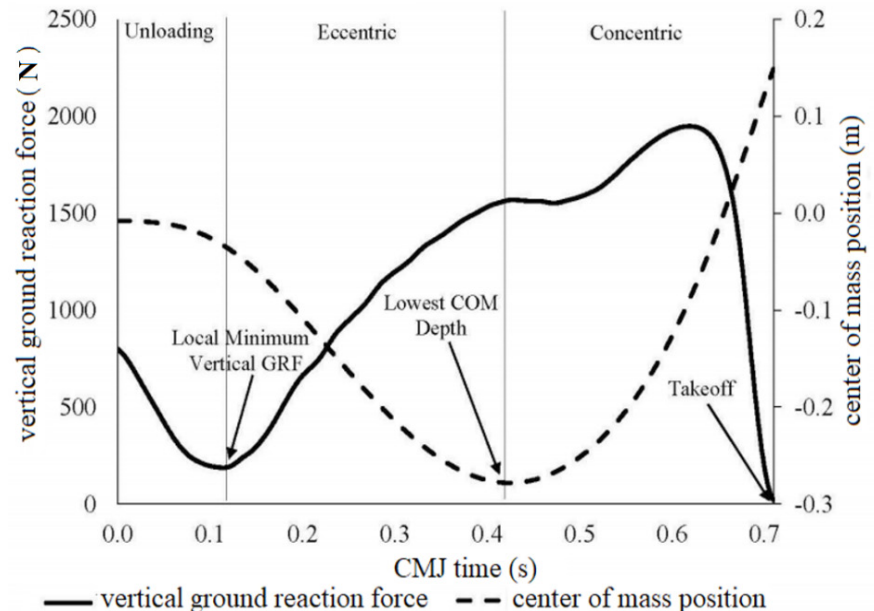

— vertical ground reaction force - - center of mass position

GRF - ground reaction force, COM - centre of mass,

CMJ - countermovement jump

Figure 1. Example of CMJ sub-phases [14].

Unloading - the unloading sub-phase; eccentric the eccentric sub-phase and respective force

development rate $(\mathrm{N} / \mathrm{s})$; concentric - the concentric sub-phase and respective force development rate $(\mathrm{N} / \mathrm{s})$.

The lowest centre of mass position depth defined the end of the eccentric sub-phase and start of the concentric sub-phase, and its displacement throughout the jump was calculated in relation to standing centre of mass

height, as suggested in previous studies [28].

Take-off defined the end of the concentric sub-phase and the start of airborne flight [14, 28]

analysis and EMG parameters were considered for validity.

\section{Statistical analysis}

Descriptive data are presented as means and standard deviations. The dependent variables of the present research were jump height, EFDR, CFDR, power, and the muscle response or electrical activity to the jump. The groups of athletes, the jump attempts, and muscle response were compared. Normal Gaussian distribution was verified by the Shapiro-Wilk's test ( $p$ values of 0.135-0.978). The information relating to the correlation between the jump height and the EFDR, CFDR, and power were analysed by intra-class correlation coefficient statistics, as described in a preceding report [18], and followed the inter-rater agreement measures: $<0.40$ for poor; $0.40-0.59$ for fair; $0.60-0.74$ for good; and 0.75-1.00 for excellent [20]. The athlete groups and CMJ attempts were compared with the use of a $2 \times 3$ between-group ANOVA and a Bonferroni post-hoc test. The Student's $t$-test was performed in comparisons of paired left vs. right side muscles. Moreover, muscle activity was compared between mus- 
cles within jump attempts with repeated measures ANOVA and a Bonferroni post-hoc test. Furthermore, the eta squared $\left(\eta^{2}\right)$ was calculated as effect size, giving 0.01 (small), 0.09 (medium), and 0.25 (large) [20]. A linear mixed model analysis was applied and included random regression effects that accounted for the influence of participants on repeated measurements, thereby enabling analysis of individual development over CMJ and EMG results. Statistical significance of the results was accepted at $p<0.05$, and all analyses were performed with the Statistical Package for the Social Sciences software (version 20.0, Chicago, USA).

\section{Ethical approval}

The research related to human use has complied with all the relevant national regulations and institutional policies, has followed the tenets of the Declaration of Helsinki, and has been approved by the Committee of Ethics in Research of the Saint Thomas University of Santiago.

\section{Informed consent}

Informed consent has been obtained from all individuals included in this study.

\section{Results}

Table 1 shows the results for the HCIV and SCBV athletes in the $3 \mathrm{CMJ}$ tests.

A large effect size and difference were verified in EFDR comparisons $\left(\mathrm{F}_{2,10}=4.948, p=0.029, \eta^{2}=\right.$ 0.275). HCIV presented higher values in the $3^{\text {rd }}$ jump than SCBV $(p=0.019)$. Concerning HCIV intra-comparisons, the $1^{\text {st }}$ jump had higher values than the $2^{\text {nd }}$ and $3^{\text {rd }}$ attempts $(p=0.05)$. Regarding SCBV intracomparisons, the $2^{\text {nd }}$ attempt showed higher values than the $3^{\text {rd }}$ jump ( $p=0.031$ ).

A large effect size and significant difference were observed in CFDR $\left(\mathrm{F}_{2,10}=7.379, p=0.032, \eta^{2}=0.747\right)$. In HCIV intra-comparisons, the $1^{\text {st }}$ jump showed higher values than the $3^{\text {rd }}$ jump ( $p=0.05$ ).

A medium effect size and difference were observed in the power produced by both volley athlete groups $\left(\mathrm{F}_{2,10}=3.543, p=0.046, \eta^{2}=0.24\right)$. The $1^{\text {st }}$ jumps obtained lower values than the $2^{\text {nd }}$ jumps ( $p=0.035$ ).

Concerning the electrical activity and muscle intercomparison, a large effect size and significant difference were observed in the $2^{\text {nd }}$ jump $\left(\mathrm{F}_{1,12}=409.499\right.$, $p \leqslant 0.001, \eta^{2}=0.972$ ). The left gluteus maximus presented lower response than the left medial gastrocnemius $(p=0.007)$.
Regarding the comparisons between left vs. right side muscles, HCIV demonstrated a main difference between the muscle response of the right vs. left gluteus maximus in the $3^{\text {rd }}$ jump $(t=3.168, d f=6, p=$ $0.019)$. No other effects were observed when comparing the 3 attempts of HCIV vs. SCBV in jump height, EFDR, CFDR, or intra- or inter-muscle electrical activity ( $p>0.05$ for all with no effect comparisons).

\section{Discussion}

A surface change can biomechanically modify athletic movements [3]. Thus, the present study aimed to compare power, height, EFDR and CFDR of 3 sequential attempts of jumps, with 1 minute of rest, verifying the electrical activity of the gluteus maximus, rectus femoris, vastus medialis, and medial gastrocnemius muscles of left and right lower limbs across the EMG during the CMJ in SCBV and HCIV athletes. This information brings new knowledge of motor actions and muscle response of the enumerated muscles during CMJ. Our analysis demonstrated ca. $70 \%$ of good or excellent intra-class correlations between attempts by both groups: SCBV and HCIV. Regarding comparisons, in the $3^{\text {rd }}$ jump HCIV presented higher EFDR values than SCBV. In addition, the $1^{\text {st }}$ jump by HCIV players had higher EFDR and CFDR than the $3^{\text {rd }}$ jump, and the $2^{\text {nd }}$ jump by SCBV presented lower values than the $3^{\text {rd }}$ jump. As for the muscle electrical activity in the $2^{\text {nd }}$ jump, the left gluteus muscle presented lower activity than the left medial gastrocnemius for SCBV and HCIV, with statistical tests as a criterion of lower and higher activity of the muscle in CMJ. According to previous authors [29], the following criteria would indicate power transfer: when the power at the proximal joint decreases, the power at the distal joint increases; then the EMG activity of the monoarticular muscles at the proximal joint continues and the biarticular muscles are activated. The present study did not measure power in the joints of lower extremities. However, preceding research suggested that concentric activation of the gastrocnemius during $\mathrm{CMJ}$ was thought to describe the power transfer from the knee extensors to the ankle joint [29]. Previous reports indicated that the design of medial gastrocnemius (a high percentage of fast-twitch muscle fibres, large angles of pinnation and short length of the fibres, long tendons, and the location about the ankle and knee joints) seemed to be well suited for transferring mechanical energy between the ankle and knee at fast speeds of locomotion [29, 30].

HCIV athletes demonstrated higher electrical ac- 
Table 1. Description and analysis of the 3 CMJs

\begin{tabular}{|c|c|c|c|c|c|c|c|}
\hline \multirow{2}{*}{ Variable } & \multirow{2}{*}{ Group } & \multirow{2}{*}{$\frac{1^{\text {st }} \mathrm{CMJ}}{\text { Mean } \pm S D}$} & \multirow{2}{*}{$\frac{2^{\text {nd }} C M J}{\text { Mean } \pm S D}$} & \multirow{2}{*}{$\frac{3^{\text {rd }} \mathrm{CMJ}}{\text { Mean } \pm S D}$} & \multicolumn{3}{|c|}{ ICC } \\
\hline & & & & & $1^{\text {st }}$ vs. $2^{\text {nd }}$ & $2^{\text {nd }}$ vs. $3^{\text {rd }}$ & $3^{\text {rd }}$ vs. $1^{\text {st }}$ \\
\hline \multirow{2}{*}{ CMJ height (cm) } & $\mathrm{HCIV}$ & $38.8 \pm 5.0$ & $39.9 \pm 6.3$ & $40.3 \pm 6.2$ & \multirow{2}{*}{$r=0.91$} & \multirow{2}{*}{$r=0.96$} & \multirow{2}{*}{$r=0.95$} \\
\hline & SCBV & $42.8 \pm 6.0$ & $43.2 \pm 7.2$ & $43.2 \pm 5.9$ & & & \\
\hline \multirow{2}{*}{ EFDR (N/s) } & $\mathrm{HCIV}$ & $-239.0 \pm 27.3$ & $-285.6 \pm 40.2^{\mathrm{a}}$ & $-270.2 \pm 31.6^{\mathrm{a}, \&}$ & \multirow{2}{*}{$r=0.50$} & \multirow{2}{*}{$r=0.22$} & \multirow{2}{*}{$r=0.71$} \\
\hline & SCBV & $-252.1 \pm 24.9$ & $-267.1 \pm 45.2$ & $-214.3 \pm 38.7^{\mathrm{b}}$ & & & \\
\hline \multirow{2}{*}{ CFDR (N/s) } & HCIV & $87.8 \pm 24.4$ & $89.6 \pm 25.7$ & $75.0 \pm 23.6^{\mathrm{a}}$ & \multirow{2}{*}{$r=0.87$} & \multirow{2}{*}{$r=0.66$} & \multirow{2}{*}{$r=0.70$} \\
\hline & SCBV & $83.1 \pm 35.6$ & $89.5 \pm 37.1$ & $77.8 \pm 32.2$ & & & \\
\hline \multirow{2}{*}{ Power (W) } & HCIV & $2341.8 \pm 342.3$ & $2433.9 \pm 327.2^{\mathrm{a}}$ & $2411.0 \pm 358.5$ & \multirow{2}{*}{$r=0.89$} & \multirow{2}{*}{$r=0.80$} & \multirow{2}{*}{$r=0.91$} \\
\hline & SCBV & $2160.5 \pm 188.4$ & $2302.1 \pm 255.7^{\mathrm{a}}$ & $2220.6 \pm 239.2$ & & & \\
\hline \multicolumn{8}{|c|}{ Muscle electrical activity during CMJ (\% of MVMC) } \\
\hline \multirow{2}{*}{ Right Gl } & HCIV & $31.5 \pm 13.2$ & $29.8 \pm 13.4$ & $34.7 \pm 17.2^{\$}$ & \multirow{2}{*}{$r=0.71$} & \multirow{2}{*}{$r=0.91$} & \multirow{2}{*}{$r=0.71$} \\
\hline & SCBV & $31.0 \pm 11.5$ & $27.2 \pm 9.8$ & $28.3 \pm 10.8$ & & & \\
\hline \multirow{2}{*}{ Left Gl } & HCIV & $22.8 \pm 9.7$ & $26.1 \pm 15.5^{\#}$ & $21.5 \pm 10.1$ & \multirow{2}{*}{$r=0.73$} & \multirow{2}{*}{$r=0.70$} & \multirow{2}{*}{$r=0.74$} \\
\hline & SCBV & $27.0 \pm 12.2$ & $21.6 \pm 9.0^{\#}$ & $26.0 \pm 10.9$ & & & \\
\hline \multirow{2}{*}{ Right RF } & HCIV & $33.7 \pm 10.2$ & $34.2 \pm 15.5$ & $31.4 \pm 9.5$ & $r=085$ & $r=083$ & $r=089$ \\
\hline & SCBV & $25.2 \pm 10.5$ & $21.8 \pm 9.9$ & $27.5 \pm 10.8$ & .05 & 0.03 & $r-0.09$ \\
\hline I eft $R F$ & HCIV & $28.1 \pm 4.9$ & $28.8 \pm 6.8$ & $30.2 \pm 8.3$ & & & $r=088$ \\
\hline LeIt KF & SCBV & $39.6 \pm 24.7$ & $31.3 \pm 18.3$ & $34.2 \pm 19.0$ & 0.85 & $r-0.95$ & $=0.88$ \\
\hline Bioht VM & HCIV & $39.8 \pm 9.5$ & $40.2 \pm 5.0$ & $40.4 \pm 9.6$ & & & $=054$ \\
\hline Kignt Vivi & SCBV & $30.3 \pm 14.4$ & $31.4 \pm 12.7$ & $29.9 \pm 13.2$ & 0.18 & $r=0.85$ & $=0.54$ \\
\hline Ieft VM & HCIV & $32.7 \pm 9.3$ & $34.5 \pm 10.5$ & $30.3 \pm 12.6$ & & & \\
\hline Len v n & SCBV & $37.3 \pm 18.4$ & $39.0 \pm 23.5$ & $37.3 \pm 14.1$ & $r-0.92$ & $r-0.03$ & $r-0.91$ \\
\hline Bight MG & HCIV & $30.6 \pm 7.7$ & $34.6 \pm 10.6$ & $33.5 \pm 13.9$ & & & \\
\hline Kignt VIG & SCBV & $32.2 \pm 8.9$ & $29.5 \pm 5.7$ & $30.5 \pm 9.0$ & $=0.05$ & $r-c$ & $=0.06$ \\
\hline Left MG & HCIV & $35.4 \pm 11.1$ & $41.2 \pm 11.7$ & $38.4 \pm 9.4$ & $r=0.67$ & $r=0.72$ & $r=0.57$ \\
\hline Left MIG & SCBV & $36.1 \pm 13.0$ & $35.5 \pm 9.6$ & $32.5 \pm 9.1$ & $r-0.01$ & & \\
\hline
\end{tabular}

CMJ - countermovement jump, ICC - intra-class correlation coefficient, $S D$ - standard deviation, EFDR - eccentric force development rate, CFDR - concentric force development rate, MVMC - maximum voluntary muscle contraction, Gl - gluteus maximus, RF - rectus femoris, VM - vastus medialis, MG - medial gastrocnemius, HCIV - hard court indoor volleyball, SCBV - sand court beach volleyball

${ }^{\text {a }}$ significant difference when compared with the $1^{\text {st }} \mathrm{CMJ}$

${ }^{\text {b }}$ significant difference when compared with the $2^{\text {nd }} \mathrm{CMJ}$

\& significant difference when compared with SCBV group

\# significant difference when compared with left MG in the same attempt and group

$\$$ significant difference when compared with left Gl in the same attempt and group $(p<0.05)$

tivity of the right than the left gluteus during the last jump. This specific lateralization in HCIV could be supported by preceding reports which evaluated the dissipation of loads during jumps, demonstrating that the pelvic structure generated a load dissipation of around 35\% during jumps using both legs in relation to the femoral joint and $42.9 \%$ during jumps using one leg [29]. In the same study, the knee articular and muscle structure contributed to $35.3 \%$ of the load dissipation during 2-leg jumps, while the 1-leg jump found $11.4 \%$; in the ankle structure, an average of $29.7 \%$ of load dissipation was found when the subjects jumped with 2 legs on the ground, and 45\% in jumps with 1 leg [29]. These evaluations were registered during the jump in a sagittal evaluation plane and demonstrated that the articular and muscle activity to the load dissipation was similar during 2-leg jumps, with slight relevance for the pelvic structures and knee, 
whereas the pelvic and ankle structures had major importance during 1-leg jumps [29].

The descriptive analysis of the vertical jump, EFDR, CFDR, and power observed that the SCBV athletes jumped 8.5\% higher than the HCIV players. HCIV demonstrated higher EFDR than SCBV in the last jump, and both groups (HCIV and SCBV) presented higher CFDR values in the $1^{\text {st }}$ jump than in the $3^{\text {rd }}$ jump. Although kinetic energy was not directly evaluated, it can be inferred from these data that good CFDR developed a greater amount of kinetic energy during the countermovement based on the sequential jumps, especially in the $2^{\text {nd }}$ jump, with higher values than in a preceding report [17]. First, the more rapid unloading sub-phase times coupled with a similar eccentric sub-phase times indicate that the countermovement was completed more quickly by good CFDR [17]. Since EFDR was higher in HCIV, good CFDR could be explained by the greater magnitude of the downward position in the centre of mass with velocity, which could be associated with the higher response of the right than the left gluteus during the last attempt, thereby maintaining the jump height. Conversely, the power produced in the $2^{\text {nd }}$ jump was higher than that in the $1^{\text {st }}$ attempt in both groups.

In contrast to our hypothesis about differences between muscle responses to CMJ, in the jump attempts, all muscles had similar responses for each general attempt, and this lack of differences was unexpected. This expectation was based on the presumption that the involved muscles would be more vigorously activated to purposefully execute EFDR and CFDR more rapidly and quickly terminate downward movement. Preceding reports indicate that a lack of differences in muscle responses might be due to using a self-selected arm swing [17]. It is possible that the self-selected arm swings employed by the good and poor CFDR values were characterized by different ranges of motion and/or angular velocities, thereby influencing the comparison of relative muscle responses. In addition, despite similar height and mass characteristics in SCBV and HCIV athletes, it is possible that different body compositions further challenged the present assessment of relative muscle responses, as differences in fatigue, blood flow, and hydration status could dramatically influence EMG signals [18, 19]. Even though these considerations should be addressed in subsequent evaluations, the current study indicates that relative muscle responses might not be a distinguishing feature of good in comparison with poor $\mathrm{CMJ}$ performance [7, 8].

A possible limitation of the current study was the sample, as it was restricted to international athletes with few participants, while effect sizes were used to address this limitation and aid in the interpretation of the data. In addition, it is recommended that subsequent investigations evaluate joint and/or segmental kinematic data, and it is suggested that longitudinal investigation tracking the changes of unloaded and loaded vertical jump abilities of SCBV and HCIV subjects would be ideal to characterize the development of specific physical components in determining the performance of volleyball jumps and the transition between both modalities. Another interesting concept is to test the athletes on a sand surface and compare the data of SCBV vs. HCIV. We did not make this comparison in the present article in order to increase the internal validity of the evaluations, since the use of sand could lead to a loss in the maximum contraction owing to ground irregularity.

Other studies with healthy subjects to evaluate the force, muscle work, and exit power during the vertical jump demonstrated that the vastus medialis muscle exerted major force and work during the jump accompanied by the gluteus maximus, rectus femoris, and medial gastrocnemius muscles [19, 31]. The analysis also revealed that the single-joint muscle groups contributed considerably more during the jump when compared with bi-articulated muscles, and that the adductors, abductors, and external rotators were always activated, although their mechanical response during the jump work was minor [9, 31]. In relation to CFDR, some studies have shown high correlations between high vertical jumps and CFDR in sports and reported that volleyball, soccer, and baseball usually do jumps, sprints, and movement changes in a short time space and require a lot of muscle action power, which enables great generation of concentric force per time unit, thereby improving vertical high jump $[32,33]$. This could be explained by describing that the force component is dependent on the properties of the musculotendinous system, independent from the earth reaction force [34, 35]. In summary, the current data indicate that high-level Chilean SCBV and HCIV athletes are good jumpers, as defined by EFDR, and produced greater power in a CMJ with a large effect size, with the ability to maintain similar power produced in the $2^{\text {nd }}$ jump. There was a lower value of power in the $3^{\text {rd }}$ jump, but this result did not affect the height. Therefore, this study exhibited greater correlation of height in both volleyball groups, and it seems that stratifying a sample of high-level volleyball athletes by jump height, power, and muscle responses would produce similar groups of jumpers. 


\section{Conclusions}

The present study compared power, height, EFDR, and CFDR of 3 sequential jump attempts, verifying the muscle response or electrical activity of the gluteus maximus, rectus femoris, vastus medialis, and medial gastrocnemius muscles across the EMG during CMJ in SCBV and HCIV athletes. HCIV presented higher EFDR values in the $3^{\text {rd }}$ jump than SCBV; however, it did not affect the height, and no effects were observed in the power between both groups. In the comparison of the 3 attempts, the $1^{\text {st }}$ jump by the HCIV subjects demonstrated higher EFDR and CFDR than the $3^{\text {rd }}$ jump, while the $2^{\text {nd }}$ jump in SCBV presented higher values than the $3^{\text {rd }}$ jump. Our analysis revealed more than $70 \%$ of good or excellent intra-class correlation between attempts by the SCBV and HCIV participants in height, power, and muscle response or electrical activity. This finding indicates the possibility of employing SCBV athletes for hard court volleyball, if one considers the determining action of the jump. The present results can be used by coaches and researchers in combination with the extensive knowledge of sports physiology as a means of supporting volleyball preparation in the context of high-level groups particularities.

\section{Disclosure statement}

No author has any financial interest or received any financial benefit from this research.

\section{Conflict of interest}

The authors state no conflict of interest.

\section{References}

1. Sheppard JM, Gabbett TJ, Stanganelli LC. An analysis of playing positions in elite men's volleyball: considerations for competition demands and physiologic characteristics.JStrength CondRes. 2009;23(6):18581866; doi: 10.1519/JSC.0b013e3181b45c6a.

2. Grgantov Z, Katić R, Marelić N. Effect of new rules on the correlation between situation parameters and performance in beach volleyball. Coll Antropol. 2005;29(2): 717-722.

3. Tilp M, Wagner H, Müller E. Differences in 3D kinematics between volleyball and beach volleyball spike movements. Sports Biomech. 2008;7(3):386-397; doi: 10.1080/14763140802233231.

4. Sánchez-Moreno J, Afonso J, Mesquita I, Ureña A. Dynamics between playing activities and rest time in highlevel men's volleyball. Int J Perform Anal Sport. 2016; 16(1):317-331; doi:10.1080/24748668.2016.11868889.

5. Medeiros A, Marcelino R, Mesquita I, Palao JM. Physical and temporal characteristics of under 19, under 21 and senior male beach volleyball players. J Sports Sci Med. 2014;13(3):658-665.

6. Nagano A, Komura T, Fukashiro S, Himeno R. Force, work and power output of lower limb muscles during human maximal-effort countermovement jumping. J Electromyogr Kinesiol. 2005;15(4):367-376; doi: 10.1016/j.jelekin.2004.12.006.

7. Mendez-Rebolledo G, Ramirez-Campillo R, GuzmanMuñoz E, Gatica-Rojas V, Dabanch-Santis A, Diaz-Valenzuela F. Short-term effects of Kinesio Taping on muscle recruitment order during a vertical jump: a pilot study. J Sport Rehabil. 2018;27(4):319-326; doi: 10.1123/ jsr.2017-0046.

8. Kopper B, Ureczky D, Tihanyi J. Trunk position influences joint activation pattern and physical performance during vertical jumping. Acta Physiol Hung. 2012;99(2): 194-205; doi: 10.1556/APhysiol.99.2012.2.13.

9. Bogdanovic Z, Smajic M, Jaksic D, Milosevic Z, Obradovic B, Gogic A, et al. Lumbar and abdominal muscles isometric potential in volleyball cadets. Int J Morphol. 2014;32(3):1036-1042; doi: 10.4067/S0717-950 22014000300047.

10. Saccol MF, Almeida GPL, de Souza VL. Anatomical glenohumeral internal rotation deficit and symmetric rotational strength in male and female young beach volleyball players. J Electromyogr Kinesiol. 2016;29: 121-125; doi: 10.1016/j.jelekin.2015.08.003.

11. Golant A, Christoforou D, Zuckerman JD, Kwon YW. Return to sports after shoulder arthroplasty: a survey of surgeons' preferences. J Shoulder Elbow Surg. 2012; 21(4):554-560; doi: 10.1016/j.jse.2010.11.021.

12. Seminati E, Minetti AE. Overuse in volleyball training/practice: a review on shoulder and spine-related injuries. Eur J Sport Sci. 2013;13(6):732-743; doi: 10. 1080/17461391.2013.773090.

13. Bele S, Östenberg AH, Sjöström R, Alricsson M. Experiences of returning to elite beach volleyball after shoulder injury. J Exerc Rehabil. 2015;11(4):204-210; doi: 10.12965/jer.150213.

14. Osborne NJ, Gatt IT. Management of shoulder injuries using dry needling in elite volleyball players. Acupunct Med. 2010;28(1):42-45; doi: 10.1136/aim.2009.001560.

15. Briner WW Jr, Benjamin HJ. Volleyball injuries: managing acute and overuse disorders. Phys Sportsmed. 1999;27(3):48-60; doi: 10.3810/psm.1999.03.720.

16. Harry JR, Paquette MR, Schilling BK, Barker LA, James CR, Dufek JS. Kinetic and electromyographic sub-phase characteristics with relation to countermovement vertical jump performance. J Appl Biomech. 2018;34(4):291-297; doi: 10.1123/jab.2017-0305.

17. Cormie P, McGuigan MR, Newton RU. Developing maximal neuromuscular power: Part 1 - biological basis of maximal power production. Sports Med. 2011;41(1): 17-38; doi: 10.2165/11537690-000000000-00000.

18. Sarvestan J, Cheraghi M, Sebyani M, Shirzad E, Svoboda Z. Relationships between force-time curve variables and jump height during countermovement jumps 


\section{HUMAN MOVEMENT}

F. Dal Bello et al., EMG in volleyball players

in young elite volleyball players. Acta Gymnica. 2018; 48(1):9-14; doi: 10.5507/ag.2018.003.

19. De Freitas VH, Ramos S, Bara-Filho M, Freitas DGS, Coimbra D, Cecchini R, et al. Effect of cold water immersion performed on successive days on physical performance, muscle damage, and inflammatory, hormonal, and oxidative stress markers in volleyball players. J Strength Cond Res. 2019;33(2):502-513; doi: 10.1519/ JSC.0000000000001884.

20. Field A. Discovering statistics using IBM SPSS Statistics, $4^{\text {th }}$ ed. London: SAGE Publications Ltd; 2018.

21. Willardson JM. A brief review: how much rest between sets? Strength Cond J. 2008;30(3):44-50; doi: 10.1519/ SSC.0b013e31817711a4.

22. Sole CJ, Mizuguchi S, Sato K, Moir GL, Stone MH. Phase characteristics of the countermovement jump force-time curve: a comparison of athletes by jumping ability. J Strength Cond Res. 2018;32(4):1155-1165; doi: 10.1519/JSC.0000000000001945.

23. Faulkner SH, Ferguson RA, Hodder SG, Havenith G. External muscle heating during warm-up does not provide added performance benefit above external heating in the recovery period alone. Eur J Appl Physiol. 2013;113(11):2713-2721; doi: 10.1007/s00421-0132708-6.

24. Bishop D. Warm up II: performance changes following active warm up and how to structure the warm up. Sports Med. 2003;33(7):483-498; doi: 10.2165/0000 7256-200333070-00002.

25. Silva LM, Neiva HP, Marques MC, Izquierdo M, Marinho DA. Effects of warm-up, post-warm-up, and rewarm-up strategies on explosive efforts in team sports: a systematic review. Sports Med. 2018;48(10):22852299; doi: 10.1007/s40279-018-0958-5.

26. Young WB, Behm DG. Effects of running, static stretching and practice jumps on explosive force production and jumping performance. J Sports Med Phys Fitness. 2003;43(1):21-27.

27. Hermens HJ, Freriks B, Disselhorst-Klug C, Rau G. Development of recommendations for SEMG sensors and sensor placement procedures. J Electromyogr Kinesiol. 2000;10(5):361-374; doi: 10.1016/s1050-6411 (00)00027-4.

28. McMahon JJ, Ripley N, Rej S. Effect of modulating eccentric leg stiffness on concentric force-velocity characteristics demonstrated in the countermovement jump. J Sport Sci. 2016;34(S1):S19.

29. Gregoire L, Veeger HE, Huijing PA, van Ingen Schenau GJ. Role of mono- and biarticular muscles in explosive movements. Int J Sports Med. 1984;5(6):301-305; doi: 10.1055/s-2008-1025921.

30. Prilutsky BI, Herzog W, Allinger TL. Forces of individual cat ankle extensor muscles during locomotion predicted using static optimization. J Biomech. 1997;30(10): 1025-1033; doi: 10.1016/s0021-9290(97)00068-7.

31. Yeow CH, Lee PVS, Goh JCH. An investigation of lower extremity energy dissipation strategies during single-leg and double-leg landing based on sagittal and frontal plane biomechanics. Hum Mov Sci. 2011;30(3):624635; doi: 10.1016/j.humov.2010.11.010.

32. McLellan CP, Lovell DI, Gass GC. The role of rate of force development on vertical jump performance. J Strength Cond Res. 2011;25(2):379-385; doi: 10.1519/JSC. 0b013e3181be305c.

33. Boullosa D, Abreu L, Conceição F, Cordero Y, Jimenez-Reyes P. The influence of training background on different rate of force development calculations during countermovement jump. Kinesiol. 2018;50(Suppl. 1):91-95.

34. Randell AD, Cronin JB, Keogh JW, Gill ND. Transference of strength and power adaptation to sports performance - horizontal and vertical force production. Strength Cond J. 2010;32(4):100-106; doi: 10.1519/ SSC.0b013e3181e91eec.

35. Kabaciński J, Murawa M, Fryzowicz A, Dworak LB. A comparison of isokinetic knee strength and power output ratios between female basketball and volleyball players. Hum Mov. 2017;18(3):40-45; doi: 10.1515/ humo-2017-0022. 